\title{
Les entreprises chimiques moyennes de la région lyonnaise : Quel avenir hors des grands groupes?
}

Hervé JOLY*

\section{Résumé}

À côté des grandes usines de la chimie lyonnaise qui appartiennent dès l'entre-deux-guerres à des groupes nationaux aux sièges parisiens, voire étrangers, il existe aussi dans la branche un certain nombre d'entreprises locales indépendantes, souvent anciennes, aux activités plus spécialisées. Toutes restent plus ou moins sous le contrôle des familles fondatrices, avec une adoption tardive du statut de société anonyme et un capital plutôt fermé. Mais, à partir des années 1960, la plupart de celles qui échappent à la fermeture intègrent des grands groupes.

\section{Mots-clés}

Industrie chimique. Groupes industriels. Concentration économique. Entreprises familiales. Capitalisme familial. Agglomération lyonnaise.

\begin{abstract}
In addition to the large chemical factories in Lyons that belonged particularly starting from the interwar period - to national firms based in Paris, or even to foreign firms, there were also local independent more or less specialized chemical companies that were often much older. These companies tended to remain more or less under the control of founding families, undergoing a late conversion to limited stock companies in the hands of a small group of shareowners. Starting in the 1960s, most of these small companies that had managed to survive were absorbed into the large firms.
\end{abstract}

\section{Keywords}

Chemical industry. Industrial groups. Merging businesses. Family companies. Family capitalism. Greater Lyons urban area.

\footnotetext{
*Hervé JOLY, Directeur de recherche CNRS, Laboratoire Triangle, Université de Lyon.
} 


\section{Introduction}

$\mathrm{Au} \mathrm{XX}^{\mathrm{e}}$ siècle, l'industrie chimique est largement associée à de grandes entreprises voire à des conglomérats, plus ou moins engagés dans les différentes branches de la chimie, organique et minérale, de base et de spécialités, jusqu'à la pharmacie, les plastiques, la photographie, les textiles artificiels et synthétiques, etc. Souvent issus de fusions, ils comptent plusieurs sites de production, dispersés sur le territoire national en fonction des ressources en matières premières, en énergie (charbon, hydroélectricité, etc.) ou des débouchés commerciaux. Leur contrôle est plutôt d'ordre managérial ou financier que familial. La France n'échappe pas à ce schéma, même si la concentration économique y est moins forte qu'en Allemagne ou en Grande-Bretagne - plusieurs groupes chimiques rassemblant par ailleurs d'autres activités comme le verre (SaintGobain) ou la métallurgie (Pechiney, Ugine). La spécificité nationale consiste surtout en une spectaculaire concentration de la gouvernance au sein de directions générales parisiennes, séparées des implantations industrielles souvent provinciales.

Mais l'industrie chimique ne se réduit pas à ces conglomérats. Il existe aussi de nombreuses entreprises moyennes, beaucoup moins connues, qui rassemblent souvent direction et fabrication sur un site unique. La région lyonnaise constitue un terrain favorable pour les étudier. Quelles sont leurs spécialités ? Qui les contrôle ? Comment se financent-elles? Dans quelle mesure parviennent-elles à survivre durablement à l'ombre des grands groupes ? 


\section{L'importance de l'industrie chimique dans la région lyonnaise}

L'importance de l'industrie chimique dans l'ancienne région Rhône-Alpes en général est bien connue. Celle-ci rassemble près d'un dixième des emplois, de l'ensemble des établissements et des grands établissements (plus de 100 salariés) nationaux de la branche en 1936 (cf. tableau 1). Le seul Rhône pèse pour près d'un vingtième des grands établissements, ce qui le met au cinquième rang des départements français, loin toutefois derrière la Seine (104), les Bouches-du-Rhône (43), la Seine-Maritime (30) et le Nord (29). Mais si l'on retire les activités d'huileries végétales et de distillation comptabilisées ici pour ne considérer que l'industrie plus spécifiquement chimique, le département dépasse avec 19 établissements les Bouches-du-Rhône (17), le Nord (17) et la SeineMaritime (9), seule la Seine (64) restant devant.

Tableau 1 : Part des effectifs et des établissements de la région Rhône-Alpes dans la branche des industries chimiques (4 G), 1936.

\begin{tabular}{|c|c|c|c|}
\hline & Effectifs & Établissements & $\begin{array}{c}\text { dont Éts de } \\
\text { plus de 100 } \\
\text { salariés }\end{array}$ \\
\hline Rhône-Alpes & $19259(9,2 \%)$ & $418(9,1 \%)$ & $43(9,9 \%)$ \\
\hline dont Rhône & $10358(4,9 \%)$ & $165(3,0 \%)$ & $21(4,8 \%)$ \\
\hline France & 209679 & 5454 & 434 \\
\hline
\end{tabular}

Source : Recensement industriel de 1936

En 1962, la tendance s'est encore accentuée, avec $12 \%$ des salariés de la branche et des établissements de plus de cent salariés dans la région, près de $17 \%$ même si l'on ne considère que les plus de cinq cents salariés, ce qui en fait de très loin la première région provinciale, loin derrière l'Ile-de-France certes. 
Tableau 2 : Répartition régionale des salariés et des établissements des industries chimiques (classes 35-36) en France, 1962.

\begin{tabular}{|l|c|c|c|c|}
\hline Régions & $\begin{array}{c}\text { Effectifs } \\
\text { salariés }\end{array}$ & Établissements & $\begin{array}{c}\text { dont Éts de } \\
\mathbf{1 0 0} \text { salariés } \\
\text { et plus }\end{array}$ & $\begin{array}{c}\text { dont Éts de } \\
\text { 500 salariés } \\
\text { et plus }\end{array}$ \\
\hline Rhône-Alpes & $\begin{array}{c}32662 \\
(12,3 \%)\end{array}$ & $\begin{array}{c}481 \\
(8,8 \%)\end{array}$ & $\begin{array}{c}66 \\
(12,0 \%)\end{array}$ & $\begin{array}{c}14 \\
(16,9 \%)\end{array}$ \\
\hline Région parisienne & 111792 & 2399 & 260 & 29 \\
\hline Nord & 19335 & 389 & 39 & 11 \\
\hline $\begin{array}{l}\text { Provence-Côte- } \\
\text { d'Azur-Corse }\end{array}$ & 15294 & 378 & 31 & 2 \\
\hline Haute-Normandie & 10171 & 131 & 21 & 5 \\
\hline Picardie & 10260 & 126 & 21 & 4 \\
\hline Aquitaine & 6733 & 297 & 17 & 1 \\
\hline Centre & 5085 & 154 & 15 & 0 \\
\hline Lorraine & 9175 & 104 & 13 & 6 \\
\hline Autres régions (12) & 44794 & 1153 & 68 & 11 \\
\hline France & 265301 & 5458 & 551 & 83 \\
\hline
\end{tabular}

Source: INSEE, Recensement de l'industrie 1963. Résultats pour 1962, Série Structures, vol. IV, Tous établissements, résultats par région, Paris, Imprimerie nationale, 1967.

À l'intérieur de la région, les données départementales de 1936 montrent une concentration des grands établissements dans l'Isère, la Savoie et surtout le Rhône, la Loire pourtant très industrialisée étant en revanche presque dépourvue d'industrie chimique. 
Tableau 3 : Répartition départementale des grands établissements des industries chimiques, 1931 et 1936.

\begin{tabular}{|c|c|c|c|c|}
\hline \multirow{2}{*}{ Départements } & \multicolumn{2}{|c|}{ Éts 101 à 500 salariés } & \multicolumn{2}{c|}{ Éts + 500 salariés } \\
\cline { 2 - 5 } & 1931 & 1936 & 1931 & 1936 \\
\hline Ain & 3 & 1 & 0 & 0 \\
\hline Ardèche & 1 & 1 & 0 & 0 \\
\hline Drôme & 1 & 0 & 0 & 0 \\
\hline Isère & 7 & 7 & 1 & 1 \\
\hline Loire & 5 & 1 & 0 & 0 \\
\hline Rhône & $\mathbf{2 1}$ & $\mathbf{1 8}$ & $\mathbf{4}$ & $\mathbf{3}$ \\
\hline Savoie & 10 & 9 & 0 & 0 \\
\hline Haute-Savoie & 2 & 2 & 0 & 0 \\
\hline Total Rhône-Alpes & 50 & 43 & 5 & 4 \\
\hline
\end{tabular}

Au-delà de ces données statistiques, la récupération des listes nominatives des recensements effectués par la Statistique générale de la France pour la région Rhône-Alpes a permis, pour les années 1931 et $1936^{1}$, une exceptionnelle identification des établissements de plus de 100 salariés concernés, sauf pour le Rhône où la liste a dû être reconstituée par d'autres sources ${ }^{2}$. On constate ainsi que, sans surprise, dans les territoires alpins (Savoie, Haute-Savoie, parties

\footnotetext{
${ }^{1}$ Sous la forme de listes manuscrites retrouvées à la délégation régionale de l'INSEE à Lyon par François Robert (Centre Pierre Léon d'histoire économique et sociale, aujourd'hui ingénieur de recherche au Laboratoire Triangle). Ces listes fournissent également les effectifs pour le recensement de 1926, mais seulement pour les établissements atteignant encore cent salariés en 1931. Elles n'ont, à notre connaissance, jamais été retrouvées pour d'autres régions françaises.

${ }^{2}$ Un peu décalées dans le temps toutefois, puisqu'elles reposent pour l'essentiel sur les archives de la Production industrielle sous l'Occupation, à l'échelon régionale fonds $130 \mathrm{~W}$ des archives départementales (AD) du Rhône, cote 138, 139 et 141 en particulier - et nationale - fichier des établissements de l'Office central de répartition des produits industriels, Archives nationales, Pierrefitte-sur-Seine, F12/9422 à 9548.
} 
montagneuses de l'Ain et de l'Isère), tous les établissements relèvent de l'électrochimie, avec de nombreuses usines fabriquant en particulier du carbure de calcium ou des produits chlorés apparues au tournant du $\mathrm{XIX}^{\mathrm{e}}$ et $\mathrm{du} \mathrm{XX}^{\mathrm{e}}$ siècles à la suite de l'explosion de l'hydro-électricité. Dans la Loire, les rares établissements qui soient rattachés, en 1931 surtout, aux industries chimiques concernent des usines à gaz (2) et des fabriques de crayon dans la région roannaise (2), seule la fabrique d'ammoniaque des Produits chimiques de Roche-la-Molière (113 salariés en 1931, 10 en 1936 seulement) relevant de la branche stricto sensu.

Il n'y a que le Rhône - qui se réduit d'ailleurs à l'agglomération lyonnaise, à laquelle on peut associer la fabrique d'eau de javel Cotelle \& Foucher à Sathonay-Rillieux (à l'époque dans l'Ain) et celles de produits chimiques Gifrer \& Barbezat à Décines et de pellicules photographiques Lumière à Feyzin (à l'époque dans l'Isère) - à rassembler une grande diversité d'industries chimiques: on y trouve des fabriques de produits de base (SaintGobain, Coignet, Progil, etc.), mais aussi de produits colorants et auxiliaires textiles (CIBA, Guimet, etc.), agricoles (Silvestre), pharmaceutiques (Givaudan-Lavirotte, Laboratoires Novalis, etc.), cosmétiques (Crème Simon), photographiques (Lumière), d'entretien (Lion Noir), etc. Au-delà du rôle particulier qu'ont pu jouer des ressources locales en matières premières comme la pyrite de Saint-Bel pour la production d'acide sulfurique, ou des besoins propres à l'industrie lyonnaise de la soierie, on retrouve de nombreuses fabrications nécessaires pour approvisionner un important bassin de population, qu'il s'agisse de l'agglomération et de son arrière-pays 
rural. Les logiques de consommation sont au moins aussi importantes dans le développement de cette industrie ${ }^{3}$.

Dans la région Rhône-Alpes, la plupart des établissements de la branche relèvent de groupes nationaux comme Pechiney, Ugine, Saint-Gobain, Rhône-Poulenc, Bozel-Malétra, etc. Dans l'électrochimie alpine, l'importance des investissements requis par une croissance rapide a débouché sur un regroupement entre quelques grands acteurs. Il en est de même pour la chimie lyonnaise des produits de base : les grandes usines de Saint-Fons et de Pierre-Bénite sont exploitées par Saint-Gobain, Ugine et Rhône-Poulenc. La chimie apparaît comme une exception en matière de contrôle économique d'après une vaste étude menée sur l'ensemble des établissements industriels et commerciaux de plus de cent salariés de la région, à partir de l'exploitation du premier registre du commerce (1920$1954)^{4}$ : le modèle dominant est celui de l'entreprise locale gérant une seule usine sur place, avec une direction et un capital concentrés entre les mains d'un cercle restreint, souvent familial. Mais une autre spécificité de l'agglomération lyonnaise est que l'industrie chimique comprend également des entreprises moyennes indépendantes.

\section{Des entreprises de spécialités}

Le corpus étudié est constitué de quatorze entreprises d'origine lyonnaise, avec des activités diversifiées dans les différentes

\footnotetext{
${ }^{3}$ Laferrère Michel, "Les industries chimiques de la région lyonnaise », Revue de géographie de Lyon, 27/3, 219-256 (1952).

${ }^{4}$ Joly Hervé, Robert François (avec Giandou Alexandre), Entreprises et pouvoir économique dans la région Rhône-Alpes (1920-1954), Centre Pierre Léon d'histoire économique et sociale, Lyon, 2003. (Cahiers Pierre Léon $n^{\circ} 4$ ).
} 
spécialités chimiques. L'établissement d'origine de ces entreprises moyennes ne dépasse pas quelques centaines de salariés.

Tableau 4 : La diversité des activités des entreprises du corpus étudié.

\begin{tabular}{|c|c|c|}
\hline Entreprises & Usine-mère & Activités \\
\hline Éts Lumière & Lyon-Montplaisir & $\begin{array}{l}\text { Produits photographiques, } \\
\text { pharmaceutiques }\end{array}$ \\
\hline Éts Progil & Lyon-Vaise & Tanins, produits agricoles \\
\hline Éts Coignet & Lyon-Mermoz & Colles, gélatine, phosphore \\
\hline Chimique de Gerland & Lyon-Gerland & Goudron, bitume \\
\hline Éts Silvestre & Lyon-Gerland & Produits chimiques agricoles \\
\hline Trux-Mistral & $\begin{array}{l}\text { Collonges-au- } \\
\text { Mont-d'Or }\end{array}$ & $\begin{array}{l}\begin{array}{l}\text { Silicate de soude, sulfate de } \\
\text { cuivre }\end{array} \\
\end{array}$ \\
\hline Éts Guimet & Fleurieu-sur-Saône & Bleu d'outremer \\
\hline $\begin{array}{l}\text { Lavix Éts Burlaton \& } \\
\text { Richelmy }\end{array}$ & Villeurbanne & Bleu d'outremer \\
\hline Éts Gonnet & Villeurbanne & $\begin{array}{lll}\text { Produits } & \text { tinctoriaux } & \text { (marque } \\
\text { Idéal) } & & \\
\end{array}$ \\
\hline Éts A. Dousselin & Lyon-Vaise & $\begin{array}{l}\text { Bougies et produits d'entretien } \\
\text { (poudre NAB) }\end{array}$ \\
\hline Éts Givaudan-Lavirotte & Lyon-États-Unis & Produits pharmaceutiques \\
\hline Laboratoires Novalis & Oullins & $\begin{array}{ll}\text { Produits } & \text { pharmaceutiques } \\
\text { (spécialités) } & \end{array}$ \\
\hline Gignoux \& $\mathrm{C}^{\mathrm{ie}}$ & Neuville-sur-Saône & $\begin{array}{l}\text { Produits parfumerie, pharmacie, } \\
\text { imprimerie }\end{array}$ \\
\hline Crème Simon & Lyon-La Guillotière & Cosmétiques \\
\hline
\end{tabular}


Tableau 5 : Effectifs de l'usine-mère des entreprises chimiques du corpus.

\begin{tabular}{|c|c|c|}
\hline Entreprises & $\begin{array}{c}1942 \\
\text { (sauf indication } \\
\text { contraire) }\end{array}$ & 1962 \\
\hline Éts Lumière & 381 & s.i. \\
\hline Éts Progil & 287 & s.i. \\
\hline Éts Coignet & 110 & s.i. \\
\hline Chimique de Gerland & 145 & 134 \\
\hline Éts Silvestre & 148 & s.i. \\
\hline Trux-Mistral & 194 & s.i. \\
\hline Éts Guimet & 198 & 63 \\
\hline $\begin{array}{l}\text { Lavix Éts Burlaton \& } \\
\text { Richelmy }\end{array}$ & $93(1943)$ & s.i. \\
\hline Éts Gonnet & 101 & 107 \\
\hline Éts A. Dousselin & 207 & 26 \\
\hline Éts Givaudan-Lavirotte & $161(1943)$ & 147 \\
\hline Laboratoires Novalis & $104(1944)$ & 134 \\
\hline Gignoux \& $C^{i e}$ & $242(1943)$ & s.i \\
\hline Crème Simon & $117(1948)$ & 80 \\
\hline
\end{tabular}

Sources : AN, F12/9498 à 9502 ; ADR, 130 W138-139 pour les années 1940 ; ADR, 3171 W49 à 52 pour 1962 .

\section{Des entreprises anciennes}

La plupart des entreprises étudiées sont très anciennes, avec des origines qui remontent souvent bien avant 1914. L'antériorité complète peut toutefois être difficile à appréhender. $\mathrm{Si}$, pour certaines, on a une continuité entre les descendants d'un fondateur qui a donné son nom à l'entreprise (Coignet, Guimet, Gillet, Trux-Mistral, Lumière, etc.), d'autres cas sont plus complexes. La Société chimique de Gerland trouve ainsi son origine dans une fabrique de produits 
chimiques (vernis, noirs de fumée, distillation de goudrons, toile bitumée pour emballages, etc.) autorisée à Gerland depuis $1859^{5}$ et exploitée, sous l'appellation Cornu Cousins ${ }^{6}$, par Charles Cornu (1816-1889). Sans postérité, celui-ci cède son entreprise en 1885 à deux jeunes partenaires, un chimiste (Auguste Boissac, 21 ans) et un représentant de commerce (François Courtois, 23 ans), qui constituent une société en nom collectif. En 1903, après le décès du second, le premier continue seul l'affaire. Mais, dès 1905, il l'apporte à une société anonyme constituée, pour en reprendre l'exploitation, par un négociant lyonnais qui en devient un éphémère directeur, la SA Anc. Éts Boissac; Boissac n'en est plus qu'actionnaire minoritaire avec $28,8 \%$ et administrateur un temps. L'année suivante, la société modifie son objet et prend, à la suite de l'apport par un industriel allemand, Adolf Gentzsch, de brevets dans le domaine des caoutchoucs - pour l'enduction de câbles sous-marins (gutta percha) en particulier -, le nom de $C^{\text {ie }}$ anonyme des procédés Gentzsch (anc. Éts Boissac). Ce n'est qu'en 1912 que celle-ci adopte la raison sociale Société chimique de Gerland. En 1919, elle se renforce dans sa nouvelle activité en absorbant la Société lyonnaise de caoutchouc, qui lui apporte une seconde usine à Villeurbanne. Le capital est maintenant très dispersé entre plusieurs centaines d'actionnaires.

L'activité industrielle peut avoir une antériorité plus grande encore. D'après son inscription au registre du commerce en 1920, Auguste Dousselin (1857-1936) était «marchand de bougies et

\footnotetext{
${ }^{5}$ AD Rhône, 5M Etcl 96, Cornu, demande d'autorisation du 4 avril 1859.

${ }^{6}$ On ne trouve pourtant pas trace dans les annuaires ou les recensements d'un autre Cornu.
} 
produits de nettoyage $»$ depuis $1893^{7}$. Mais, sur son papier à en-tête utilisé en 1928 pour demander l'autorisation de transférer ses ateliers de « moulage de bougies et autres objets en cire et en acide stéarique » un peu plus loin sur le quai d'Arloing figure la mention "maison fondée en $1792^{8} »$. La fabrication de poudre à récurer (marque NAB), commercialisée depuis $1905^{9}$, n'est pas évoquée. Lorsque, en 1901, il avait demandé l'autorisation pour sa précédente implantation, son papier à en-tête était encore imprimé au nom de «F. Dumortier», avec l'ajout au tampon de la mention «A. Dousselin successeur », et il s'agissait déjà d'un transfert d'atelier, depuis la montée du Chemin neuf (quartier Saint-Jean dans le vieux Lyon) dont son prédécesseur avait obtenu l'autorisation ${ }^{10}$. Celui-ci n'était autre que son beau-frère Félix Dumortier. Les deux hommes avaient d'abord regroupé leurs activités dans une société commune constituée en août 1899; Dousselin a apporté un magasin rue de la Platière (quartier des Terreaux), Dumortier à la fois un commerce rue de la Bombarde (quartier Saint-Jean) et son usine, en se retirant de la gestion comme simple commanditaire. La société est dissoute moins d'un an plus tard et Dousselin en poursuit seul l'activité. Son beau-frère avait obtenu l'autorisation de fabrication montée du Chemin neuf en $1894^{11}$, à la

\footnotetext{
${ }^{7}$ AD Rhône, registre du commerce, greffe du tribunal de commerce de Lyon, inscription $\mathrm{n}^{\circ}$ A 5526, 8 septembre 1920.

8 AD Rhône, 5M Etcl 234, n 1267, lettre au préfet du Rhône du 27 février 1928.

${ }^{9}$ Nadine Halitim-Dubois, «Usines de bougies dite Dousselin puis Usine de détergents dite Nab actuellement parc d'activité », Région Rhône-Alpes, Inventaire général du patrimoine culturel, patrimoine.rhonealpes.fr.

${ }^{10}$ AD Rhône, 5M Etcl 1949, atelier d'Auguste Dousselin, lettre au préfet du Rhône du 4 janvier 1901.

${ }^{11}$ AD Rhône, 5M Etcl 127, Fabrique de cierges Félix Dumortier, autorisation du 21 décembre 1894.
} 
suite du transfert du premier atelier rue de la Bombarde dont sa mère veuve avait elle-même obtenu l'autorisation en 1862, pour une fabrication qui existait depuis « de longues années à la maison ${ }^{12}$ ». Dumortier était effectivement fils et petit-fils de «négociant», sans que sa généalogie directe ne permette de remonter jusqu'en $1792^{13}$.

Autre exemple d'origine complexe, l'appellation Laboratoires Novalis apparaît pour la première fois en 1921 comme dénomination commerciale d'une société en nom collectif É. Dupraz \& $C^{\text {ie }}$ qui associe deux pharmaciens et un industriel pour la fabrication « de tous appareils et produits hygiéniques, [...] d'accessoires de pharmacie et médicaments en tube étain, $[\ldots]$ des produits et spécialités connues sous le nom de Élixir Guillet et Laucodragette ${ }^{14}$. Mais ces activités correspondent en fait à la réunion des affaires antérieures respectives des associés, l'industriel Louis Montchanin et les pharmaciens Émile Dupraz et Joseph Laurençon. Celle du second, qui marque la continuité de la raison sociale, avait, d'après son objet - « achat et vente de tous instruments de chirurgie et tous articles dits accessoires de pharmacie ainsi que fabrication de quelques-uns de ces articles »-, une dimension industrielle. Elle-même reposait sur le rachat à une veuve d'un fonds de commerce de bandagisterie existant depuis les années $1870^{15}$.

\footnotetext{
${ }^{12}$ AD Rhône, 5M Etcl 98, $\mathrm{V}^{\mathrm{ve}}$ Dumortier, lettre au sénateur du Rhône, 26 mars 1862.

${ }^{13}$ Son grand-père Antoine Marie, commis-négociant à son mariage en 1817, est le fils d'un propriétaire rural, né à Pollionay (Rhône) en 1788.

${ }^{14}$ AD Rhône, 6Up1/343, acte de transformation, 7 juin 1921.

${ }^{15}$ AD Rhône, 6Up1/336, acte dissolution, 31 janvier 1921. Le fonds exploité sous le nom Ravut-Charles apparaît dans l'édition 1875 de l'annuaire du Rhône ; à son mariage en 1871, Jacques Henri Ravut était encore déclaré représentant de commerce. Le commerce est poursuivi après son décès en 1884 par sa veuve
} 
Enfin, le fondateur d'une entreprise peut être déjà engagé dans d'autres affaires industrielles: Georges Gignoux, lorsqu'il crée en 1934, à l'âge de 52 ans, l'entreprise Gignoux \& $C^{\text {ie }}$ pour la fabrication « de tous produits chimiques, vétérinaires, agricoles, de parfumerie et d'hygiène », était déjà associé de l'entreprise chimique de Décines Gignoux Frères \& Barbezat.

L'ancienneté d'une entreprise, entendue ici comme le démarrage de l'activité industrielle (cf. tableau 6), comporte donc une certaine dimension arbitraire.

Tableau 6 : Ancienneté de l'activité industrielle des entreprises du corpus.

\begin{tabular}{|l|c|}
\hline Entreprises & Démarrage de l'activité industrielle \\
\hline Éts Coignet & 1818 \\
\hline Éts Guimet & 1826 \\
\hline Éts Gillet / Progil & 1838 (teinture) \\
\hline Chimique de Gerland & 1859 \\
\hline Crème Simon & 1860 \\
\hline Éts A. Dousselin & $<1861 ?$ \\
\hline Éts Lumière & 1882 \\
\hline Éts Silvestre & 1890 \\
\hline Éts Givaudan-Lavirotte & 1891 \\
\hline Lavix Éts Burlaton \& Richelmy & 1907 \\
\hline Laboratoires Novalis & $<1913 ?$ \\
\hline Éts Gonnet & 1917 \\
\hline Gignoux \& C & 1934 \\
\hline
\end{tabular}

\section{L'ascension sociale des fondateurs}

Philomène Antoinette Charles, remariée ensuite à un receveur des contributions indirectes entre-temps décédé également. 
Les fondateurs sont souvent d'origine rurale, de la région au sens large, jusqu'à la Haute-Saône pour Antoine Lumière; il est remarquable qu'ils sont une minorité à être nés dans l'agglomération lyonnaise (quatre seulement), ce qui montre le renouvellement de la population urbaine. Les origines sociales sont - à quelques exceptions près comme Jean François Coignet, Jean Baptiste Guimet ou, bien sûr, Georges Gignoux, issu d'une famille déjà engagée dans l'industrie plutôt modestes, dans le monde du petit commerce ou de l'agriculture en particulier. L'industrie apparaît bien comme un facteur d'ascension sociale.

Les fondateurs sont plutôt des autodidactes, sauf pour les entreprises pharmaceutiques (Xavier Givaudan, Émile Dupraz) que la législation oblige à avoir pour gérants des pharmaciens de métier ${ }^{16}$. La seule autre exception est Jean-Baptiste Guimet, polytechnicien (promotion 1813), qui avait même passé le concours une deuxième année pour entrer avec un meilleur rang $\left(55^{\mathrm{e}}\right)$, et qui est sorti très bien classé $\left(6^{\mathrm{e}}\right)$, ce qui lui a permis d'intégrer le corps militaire des Poudres ${ }^{17}$.

L'École de chimie industrielle de Lyon fondée en 1883 au sein de la faculté des sciences ne joue aucun rôle dans la formation de la génération des fondateurs. Seuls des représentants de la troisième

\footnotetext{
${ }^{16}$ Chauveau Sophie, L'Invention pharmaceutique. La pharmacie française entre l'État et la société au XX siècle, Institut d'édition Sanofi-Synthélabo, Paris, 1999, p. 25 et 212. L'obligation ne vaut pas pour les accessoires pharmaceutiques comme les bandages (activité dont sont issus les Laboratoires Novalis).

${ }^{17}$ Informations disponibles dans la base La Famille polytechnicienne, consultable sur le site de la bibliothèque de l'École polytechnique, htpps://biblialeph.polytechnique.fr.
} 
génération (Edmond Gillet et Paul Gillet, Michel Trux) en ont été élèves.

Tableau 7 : Origine géographique et sociale des fondateurs des entreprises.

\begin{tabular}{|c|c|c|c|c|}
\hline Entreprises & Fondateur & $\begin{array}{l}\text { Année de } \\
\text { naissance }\end{array}$ & Lieu & $\begin{array}{c}\text { Profession } \\
\text { du père }\end{array}$ \\
\hline Éts Coignet & $\begin{array}{c}\text { Jean-François } \\
\text { Coignet }\end{array}$ & 1793 & $\begin{array}{l}\text { Saint- } \\
\text { Étienne }\end{array}$ & $\begin{array}{l}\text { marchand } \\
\text { armurier }\end{array}$ \\
\hline Éts Guimet & $\begin{array}{l}\text { Jean Baptiste } \\
\text { Guimet }\end{array}$ & 1795 & $\begin{array}{l}\text { Voiron } \\
\text { (Isère) }\end{array}$ & $\begin{array}{c}\text { ingénieur } \\
\text { des Ponts } \\
\text { et } \\
\text { Chaussées }\end{array}$ \\
\hline Éts Gillet / Progil & François Gillet & 1813 & $\begin{array}{c}\text { Bully } \\
\text { (Rhône) }\end{array}$ & agriculteur \\
\hline Chimique de Gerland & Charles Cornu & 1816 & $\begin{array}{l}\text { Jallieu } \\
\text { (Isère) }\end{array}$ & dessinateur \\
\hline Crème Simon & $\begin{array}{l}\text { Benoit Joseph } \\
\text { Simon }\end{array}$ & 1815 & $\begin{array}{l}\text { Charlieu } \\
\text { (Loire) }\end{array}$ & $\begin{array}{l}\text { marchand } \\
\text { épicier }\end{array}$ \\
\hline Trux-Mistral & Fleury Trux & 1834 & Villeurbanne & charcutier \\
\hline Éts Lumière & $\begin{array}{l}\text { Antoine } \\
\text { Lumière }\end{array}$ & 1840 & $\begin{array}{l}\text { Ormoy } \\
\text { (Haute- } \\
\text { Saône) }\end{array}$ & vigneron \\
\hline Éts Silvestre & $\begin{array}{l}\text { Claude } \\
\text { Silvestre }\end{array}$ & 1866 & $\begin{array}{l}\text { Le Bois- } \\
\text { d'Oingt } \\
\text { (Rhône) } \\
\end{array}$ & agriculteur \\
\hline Éts Givaudan-Lavirotte & $\begin{array}{c}\text { Xavier } \\
\text { Givaudan }\end{array}$ & 1867 & $\begin{array}{c}\text { Caluire-et- } \\
\text { Cuire } \\
\text { (Rhône) }\end{array}$ & $\begin{array}{c}\begin{array}{c}\text { voyageur } \\
\text { de } \\
\text { commerce }\end{array} \\
\end{array}$ \\
\hline $\begin{array}{l}\text { Lavix Éts Burlaton \& } \\
\text { Richelmy }\end{array}$ & Pierre Burlaton & 1859 & Lyon & $\begin{array}{l}\text { commis- } \\
\text { négociant }\end{array}$ \\
\hline Laboratoires Novalis & Émile Dupraz & 1865 & $\begin{array}{l}\text { Chaley } \\
\text { (Ain) }\end{array}$ & $\begin{array}{c}\text { garde- } \\
\text { champêtre }\end{array}$ \\
\hline Éts Gonnet & Louis Gonnet & 1880 & $\begin{array}{c}\text { Morestel } \\
\text { (Isère) }\end{array}$ & aubergiste \\
\hline Gignoux \& $C^{i e}$ & $\begin{array}{l}\text { Georges } \\
\text { Gignoux }\end{array}$ & 1882 & Lyon & industriel \\
\hline
\end{tabular}

\section{L'adoption tardive de la société anonyme}

Ces entreprises moyennes ont longtemps conservé un cadre fermé. La plupart ont d'abord été exploitées en entreprises individuelles, sans distinction avec la personnalité physique de leur 
propriétaire. La création d'une société commerciale est rarement concomitante, et lorsqu'elle l'est, c'est, en présence de deux ou trois fondateurs, dans le cadre d'une société en nom collectif (SNC) qui engage totalement la responsabilité de leurs associés (Gillet, Givaudan-Lavirotte, Laboratoires Novalis). Seule Gignoux \& $C^{\text {ie }}$ est constituée directement en SARL, après l'introduction tardive (1925) de ce statut en droit français.

L'entreprise individuelle peut se prolonger entre plusieurs générations, sur plusieurs décennies, plus d'un siècle dans le cas des Éts Guimet, la veuve du petit-fils du fondateur continuant même l'exploitation huit ans ainsi. Mais l'hypothèse la plus fréquente est que l'apport en société se fasse dans le cadre de la succession du fondateur, avant ou après sa mort, entre ses différents enfants. Antoine Lumière le fait dès l'âge de 44 ans, en 1884, avec ses deux fils Auguste et Louis, âgés respectivement de 22 et 20 ans. Fleury Trux a, lui, 58 ans lorsqu'il associe son fils Antoine, 28 ans, en 1892 à ses affaires et Pierre Burlaton en a 63 lorsqu'il le fait en 1922 dans le cadre de la Société des bleus d'outre-mer Lumyère avec ses deux fils et son gendre. En revanche, la société Crème Simon est créée après le décès du fondateur, entre ses quatre fils. Au décès de Louis Gonnet à 48 ans en 1928, sa veuve reprend seule l'affaire éponyme, et ce n'est qu'en 1945, à 67 ans, qu'elle associe ses quatre enfants entre-temps tous majeurs.

À l'exemple de Dumortier et Dousselin ${ }^{18}$, la création d'une société peut même être provisoire et réversible, l'un des associés

\footnotetext{
${ }^{18}$ Une première société en nom collectif avait été fondée en novembre 1898 entre Dumortier et un autre négociant, Jules Barthe, avant d'être dissoute en juillet suivant
} 
reprenant ensuite durablement l'exploitation en entreprise individuelle. Il en est de même pour François Gillet, avec l'association éphémère avec un autre teinturier en 1838, puis avec plusieurs associations successives avec ses différents beaux-frères Pierron à partir de 1844, avant qu'il ne poursuive l'exploitation seul en 1861 jusqu'à la transmission progressive à ses fils à partir de $1869^{19}$.

La transformation en société par actions est en général encore plus tardive. Seuls Silvestre en commandite par actions (SCA) et Dousselin en société anonyme (SA) l'ont fait directement. Le nombre d'actionnaires de ces sociétés reste faible au départ, proche du minimum légal de sept introduit en 1867. Il en est de même lorsque le statut est adopté par transformation de sociétés de personnes. Grâce aux seules actions en rémunération de leurs apports en nature, les fondateurs gardent en général le contrôle. Malgré l'adoption formelle du statut de SCA en 1855, les trois fils Coignet sont restés seuls associés en nom collectif. Ce n'est que contraints par la loi de 1867 qu'ils ont introduit l'année suivante des commanditaires très minoritaires $(1,3 \%)$. Les Lumière père et fils reçoivent ainsi 5600 des 6000 actions $(93,3 \%)$ de la SA formée en 1892, les vingt-sept souscripteurs contre espèces se partageant le reste. Il en est de même pour Claude Silvestre en 1930 qui, entre 7000 actions d'apport et 820 souscrites sur 8500 , détient $92 \%$ du capital de la SCA constituée. Seuls Xavier Givaudan et son associé Antonin Trouillat sont moins bien lotis en 1899 avec seulement 1000 actions d'apport sur 6000

pour des raisons liées à la santé du premier, ce qui explique qu'il se contente ensuite d'un statut de commanditaire de son beau-frère.

${ }^{19}$ Joly H., Les Gillet de Lyon. Fortunes d'une grande famille industrielle (18382015), Droz, Genève, 2015, chapitre 1. 
$(16,7 \%)$ du capital de la SCA Givaudan Trouillat \& $C^{\text {ie }}$ (future Givaudan Lavirotte \& $C^{\mathrm{ie}}$ ) dont ils conservent la gérance, le reste étant partagé entre de nombreux commanditaires parmi lesquels on trouve d'autres pharmaciens, ainsi que les frères Auguste et Louis Lumière.

Tableau 8 : Créations des entreprises et formes d'exploitation en sociétés.

\begin{tabular}{|c|c|c|c|c|}
\hline Entreprises & $\begin{array}{l}\text { Création } \\
\text { entreprise }\end{array}$ & $\begin{array}{l}\text { Première } \\
\text { création } \\
\text { société } \\
\text { (nature) }\end{array}$ & $\begin{array}{c}\text { Formation } \\
\text { société par } \\
\text { actions }\end{array}$ & $\begin{array}{c}\text { Nombre } \\
\text { d'actionnaires } \\
\text { de la première } \\
\text { société par } \\
\text { action }\end{array}$ \\
\hline Éts A. Dousselin & 1901 & 1898 (SNC) & 1932 & 7 \\
\hline Éts Coignet & 1818 & $1822(\mathrm{SNC})$ & $\begin{array}{c}1855(\mathrm{SCA}) / \\
1914(\mathrm{SA})\end{array}$ & 3 \\
\hline Éts Guimet & 1826 & $\begin{array}{c}1928 \\
(\mathrm{SARL})\end{array}$ & néant & l \\
\hline $\begin{array}{l}\text { Éts Gillet / } \\
\text { Progil }\end{array}$ & $\begin{array}{c}1838 \\
\text { (teinture) }\end{array}$ & $1838(\mathrm{SNC})$ & 1918 & 7 \\
\hline $\begin{array}{l}\text { Chimique de } \\
\text { Gerland }\end{array}$ & 1859 & 1886 (SNC) & 1905 & 8 \\
\hline Crème Simon & 1860 & $1906(\mathrm{SNC})$ & 1926 & 10 \\
\hline Trux-Mistral & $\approx 1865$ & 1892 (SNC) & 1933 & 7 \\
\hline Éts Lumière & 1882 & 1884 (SNC) & 1892 & 30 \\
\hline Éts Silvestre & 1890 & 1930 (SCA) & $\begin{array}{c}1930 / \\
1931(\mathrm{SA})\end{array}$ & 14 \\
\hline $\begin{array}{l}\text { Éts Givaudan- } \\
\text { Lavirotte }\end{array}$ & 1891 & 1891 (SNC) & $\begin{array}{c}1899(\mathrm{SCA}) / \\
1915(\mathrm{SA})\end{array}$ & 242 \\
\hline $\begin{array}{l}\text { Lavix Éts } \\
\text { Burlaton \& } \\
\text { Richelmy }\end{array}$ & 1907 & 1922 (SCS) & 1938 & 8 \\
\hline $\begin{array}{l}\text { Laboratoires } \\
\text { Novalis }\end{array}$ & 1913 & $1913(\mathrm{SNC})$ & 1938 & 14 \\
\hline Éts Gonnet & 1917 & 1945 (SNC) & 1965 & s.i. \\
\hline Gignoux \& $\mathrm{C}^{\mathrm{ie}}$ & 1934 & $\begin{array}{c}1934 \\
\text { (SARL) }\end{array}$ & 1948 & 22 \\
\hline
\end{tabular}

\section{Un contrôle familial resté dominant}

La transformation plus ou moins tardive en société par actions ne débouche pas nécessairement sur des appels fréquents aux 
actionnaires pour apporter de nouveaux capitaux (voir tableau 9 ciaprès). Et ces appels peuvent rester dans un cadre très restreint, à l'exemple de Novalis où le nombre de souscripteurs atteint un maximum de 18 en 1948, ou, pour une souscription unique, de Dousselin (9) ou Burlaton \& Richelmy (2). À l'inverse, dans d'autres sociétés entrées en bourse, le nombre de souscripteurs peut attendre plusieurs centaines (Chimique de Gerland, Givaudan-Lavirotte) voire milliers (Coignet, Lumière et surtout Progil).

Il n'y a cependant pas de lien strict entre ouverture du capital et préservation du contrôle par les fondateurs ou leurs descendants. Dans des sociétés non cotées comme Crème Simon, Dousselin ou Lavix jusqu'en 1942, les familles assurent l'intégralité des apports et conservent le contrôle de l'entreprise. Les conseils d'administration restent exclusivement composés de descendants. Chez Crème Simon, le petit-fils (1942) et l'arrière-petit-fils (1957) du fondateur sont successivement nommés PDG. Chez Dousselin, la fille veuve d'Auguste préside le conseil d'administration et, en 1959, le petit-fils prend le relais.

Dans d'autres sociétés non cotées, des appels fermés débouchent néanmoins sur l'arrivée de nouveaux actionnaires. En octobre 1931, pour venir au secours des Éts Silvestre frappés par la crise, Alais, Froges \& Camargue (AFC, future Pechiney) souscrit la quasi-intégralité des 2,5 millions de francs apportés à une nouvelle $\mathrm{SA}$, quatre millions étant partagés à titre d'apports en nature entre l'ancienne société et AFC, qui obtient ainsi au moins $69 \%$ du capital, et probablement plus, le fondateur Claude Silvestre ayant déjà quitté l'entreprise depuis janvier. En 1948, une filiale d'AFC souscrit à 
nouveau la quasi-totalité des actions. Silvestre n'est plus une entreprise indépendante; les représentants d'AFC dominent son conseil d'administration. De même, chez Gignoux \& $\mathrm{C}^{\mathrm{ie}}$, la participation familiale, qui n'était déjà plus dans l'ancienne SARL, avec les trois fils du fondateur ${ }^{20}$, que de $50,01 \%$ - les quatre filles ayant vendu leurs actions en 1947 -, ne pèse plus que $5 \%$ d'une souscription doublant le capital l'année suivante. Le groupe Schneider, avec sa filiale bancaire Union européenne industrielle et financière, prend le contrôle de l'entreprise. Ses représentants obtiennent deux sièges sur trois au conseil d'administration, même si l'aîné des trois frères, Paul Gignoux, reste PDG.

À l'inverse, chez Progil, même après l'entrée en bourse, l'explosion du nombre d'actionnaires n'empêche pas la famille Gillet de conserver le contrôle de l'entreprise, avec des participations qui restent supérieures à $40 \%$ dans des émissions pourtant nombreuses (voir tableau 10 ci-après). La fortune dont dispose la famille, engagée dans de nombreuses autres affaires industrielles, lui permet de suivre et de garder le contrôle exclusif du conseil d'administration, même si la direction générale revient à un manager, Maurice Brulfer.

De même, chez Givaudan-Lavirotte, le fondateur Xavier Givaudan reste durablement l'actionnaire dominant, avec $53 \%$ (1939), $35 \%$ (1946) et $44 \%$ (1948) des souscriptions successives, un manager devenant PDG en 1948. En revanche, chez Coignet, la participation familiale tombe dès 1921 à $10,3 \%$. Elle remonte à près d'un quart en 1928, pour la création de 4000 actions à droit de vote

\footnotetext{
${ }^{20}$ Le fondateur avait cédé l'ensemble de ses parts de la SARL à ses sept enfants en 1942 à titre de partage très anticipé - il ne décède qu'en 1963.
} 
plural destinées à protéger le capital, mais cette souscription marque aussi l'arrivée d'un grand groupe, Kuhlmann, avec 19,8\%, et ces actions sont interdites par une loi en 1933. Lors des émissions suivantes dont les répartitions sont connues, si la famille est encore présente à la hauteur de près de $10 \%$ en 1945 , sa participation devient dérisoire en 1957. Kuhlmann est devenu l'actionnaire dominant, à la hauteur de plus d'un quart. Les Coignet se sont entre-temps retirés de la présidence et n'ont plus que deux sièges sur douze au conseil d'administration. Chez Lumière, la famille fondatrice ne souscrit plus que $2,9 \%$ des actions ordinaires émises en 1928 ; elle est largement dépassée par le groupe financier parisien Rivaud (52\%), mais elle se rattrape avec des actions à vote plural dont elle se réserve 83,3\%. Leur suppression effective en 1935 affaiblit sa position. En 1950, l'apport des Lumière n'est plus que 3,2\%, et ils sont devancés à nouveau par le groupe Rivaud (11\%) et par le Crédit lyonnais (8\%). Mais ils gardent la présidence-direction générale avec Henri Lumière et quatre sièges sur cinq au conseil d'administration.

La Société chimique de Gerland est la seule entreprise à avoir un capital dispersé, sans actionnaire dominant, l'apporteur de l'entreprise en SA Auguste Boissac ayant depuis longtemps disparu. Les dirigeants polytechniciens, Courbier père et fils, assurent un contrôle managérial avec une minorité croissante des actions (4,6 \% en $1920,16,6 \%$ en 1947$)$. 
Tableau 9 : Sociétés par actions et apports en capitaux.

\begin{tabular}{|c|c|c|c|}
\hline Entreprises & $\begin{array}{l}\text { Formation } \\
\text { société par } \\
\text { actions }\end{array}$ & $\begin{array}{c}\text { Émissions d'actions } \\
\text { (avec nombre de } \\
\text { souscripteurs) }\end{array}$ & $\begin{array}{c}\text { Année } \\
\text { cotation } \\
\text { en bourse }\end{array}$ \\
\hline Éts Coignet & $\begin{array}{c}1855 / 1914 \\
(\mathrm{SA})\end{array}$ & $\begin{array}{c}1918(250), 1921(648), 1928 \\
(242), 1929(459), 1945(1268), \\
1947 \text { (s.i.), } 1948(2200), 1950 \\
(1878), 1953(2470), 1957 \\
(2745)\end{array}$ & 1917 \\
\hline $\begin{array}{l}\text { Givaudan- } \\
\text { Lavirotte }\end{array}$ & $\begin{array}{l}1899 / 1915 \\
(\mathrm{SA})\end{array}$ & $\begin{array}{c}1915(82), 1918(140), 1920 \\
(160), 1939(87), 1946(390) \\
1948(499)\end{array}$ & 1901 \\
\hline Éts Lumière & 1892 & $\begin{array}{c}1928(>1000), 1947 \\
(5557), 1950(2832), 1962 \\
(2954)\end{array}$ & 1912 \\
\hline $\begin{array}{l}\text { Chimique de } \\
\text { Gerland }\end{array}$ & 1905 & $\begin{array}{c}1906(78), 1907(217), 1916 \\
(348), 1921(542), 1947(1153)\end{array}$ & 1912 \\
\hline Éts Gillet / Progil & 1918 & $\begin{array}{c}1920(5), 1923(10), 1925(1), 1931 \\
(1), 1942(27), 1948(3728), 1952 \\
(3868), 1955(4589), 1957(5237), \\
1959(6272), 1960(7287), 1961 \\
\text { (s.i.), } 1965 \text { (s.i), } 1969(10082)\end{array}$ & 1947 \\
\hline Crème Simon & 1926 & $1928(11), 1929(?)$ & 1 \\
\hline Éts Silvestre & $\begin{array}{l}1930 / 1931 \\
\text { (SA) }\end{array}$ & $1931(10), 1948(10)$ & 1 \\
\hline Éts A. Dousselin & 1932 & $1943(9)$ & 1 \\
\hline Trux-Mistral & 1933 & 1 & 1 \\
\hline $\begin{array}{l}\text { Lavix Éts } \\
\text { Burlaton \& } \\
\text { Richelmy }\end{array}$ & 1938 & $1952(2)$ & / \\
\hline $\begin{array}{l}\text { Laboratoires } \\
\text { Novalis }\end{array}$ & 1938 & $\begin{array}{c}1943 \text { (14), } 1944 \text { (13), } 1948 \text { (18), } \\
1949 \text { (1), } 1952 \text { (13), } 1965 \text { (s.i.) }\end{array}$ & / \\
\hline Gignoux \& $C^{i e}$ & 1948 & $1948(27)$ & 1 \\
\hline Éts Gonnet & 1965 & 1 & 1 \\
\hline Éts Guimet & 1965 & 1 & 1 \\
\hline
\end{tabular}

Tableau 10 : Participations de la famille Gillet aux souscriptions de Progil après l'entrée en bourse, 1948-1960²1.

\begin{tabular}{|c|c|c|c|c|c|c|}
\hline & $\mathbf{1 9 4 8}$ & $\mathbf{1 9 5 2}$ & $\mathbf{1 9 5 5}$ & $\mathbf{1 9 5 7}$ & $\mathbf{1 9 5 9}$ & $\mathbf{1 9 6 0}$ \\
\hline Participations & $52,2 \%$ & $51,7 \%$ & $40,8 \%$ & $51,2 \%$ & $48,5 \%$ & $46,5 \%$ \\
\hline
\end{tabular}

\footnotetext{
${ }^{21}$ Les données ne sont plus disponibles à partir de 1961 ; on sait seulement que, en 1965 et 1969, deux sociétés familiales souscrivent encore au moins 40,2\% et 29,9\% par compensation avec le montant de leurs comptes courants.
} 


\section{Une expansion nationale avec un ancrage régional préservé}

La majorité de ces entreprises moyennes sont restées des entreprises mono-établissement, avec une implantation industrielle unique généralement confondue avec le siège social lyonnais. Seules quatre sont devenues des entreprises avec des implantations multiples à l'échelle régionale et même nationale :

- Coignet avait, après une première implantation à Saint-Rambert au nord de Lyon, son usine historique à Baraban, à l'est ; une seconde est rachetée en 1846 un peu plus loin, route d'Heyrieux et une troisième est implantée en 1851 à Saint-Denis près de Paris. En 1914, après la fermeture de Baraban, la société absorbe deux autres entreprises qui lui apportent des usines à Grigny (Rhône), Poissy (Haute-Savoie) et Marseille l'Estaque pour la première (Mital \& $C^{\text {ie }}$ ), et à Saint-Fons pour la seconde (S. Laprévote \& $\mathrm{C}^{\mathrm{ie}}$ ). En 1925, une autre usine est rachetée à Épierre (Savoie) pour fabriquer du phosphore en utilisant l'hydroélectricité. Il n'en subsiste plus que cinq ensuite (LyonHeyrieux, Grigny, Saint-Denis, Épierre, Marseille-L'Estaque).

- Progil, après une éphémère implantation en Savoie, s'implante, pour exploiter les forêts de châtaignier des pourtours du Massif central, avec un rachat dans le Gard (1899) et une création en Dordogne (1907). Après la guerre, ce sont une quinzaine d'usines d'extraits tannants, mais aussi d'autres fabrications chimiques, qui sont reprises pour l'essentiel dans la moitié sud de la France; certaines sont fermées rapidement, d'autres conservées plus durablement, avec notamment le développement de sites importants à Clamecy (Nièvre) 
et Pont-de-Claix (Isère); s'y ajoutent des filiales et de multiples participations communes avec d'autres groupes chimiques ${ }^{22}$.

- Lumière fait, en 1903, construire une seconde usine pour la fabrication de pellicules à Feyzin, au sud de l'agglomération lyonnaise, pour éviter la pollution due aux vapeurs d'éther à Monplaisir. En 1911, la fusion avec la société parisienne Jougla apporte l'importante usine de Joinville-sur-Seine.

- en 1919, Chimique de Gerland reprend l'usine de la Société lyonnaise de caoutchouc à Villeurbanne. De nombreux sites de production s'ajoutent ensuite à Voglans (Savoie), Marseille, PortSaint-Louis-du-Rhône (Bouches-du-Rhône) et La Trinité-Victor près de Nice.

Cette expansion amène les trois premières entreprises à transférer leur siège social à Paris, Coignet dès 1872, Lumière en 1911 lors de la fusion avec Jougla et Progil en 1944, à la suite des autres affaires du groupe Gillet. Mais les directions restent, avec les familles fondatrices, plus longtemps lyonnaises; les patrons Jean Coignet (1911-1922) et Henri Lumière (1945-1955) président ainsi la chambre de commerce locale. Il n'y a pas la même rupture que pour des grandes entreprises comme la Société chimique des usines du Rhône ou Alais \& Camargue dont les directions deviennent, dès les années 1900, plus exclusivement parisiennes, avec le retrait des fondateurs lyonnais ${ }^{23}$.

\footnotetext{
22 Joly, H., Les Gillet de Lyon, op. cit., note 19, chapitre VI.

${ }^{23}$ Même si Alais \& Camargue, puis AFC conserve formellement son siège social à Lyon.
} 


\section{L'attraction des groupes comme alternative au déclin ?}

Mais, qu'elles soient restées des entreprises isolées, ou qu'elles aient constitué des groupes, ces entreprises moyennes n'ont guère conservé leur indépendance au-delà des années 1960 (voir tableau 11 ci-après). Certaines, comme Lavix, Dousselin ou Crème Simon, ont purement et simplement disparu. Beaucoup, à la suite de Silvestre dès 1931, sont passées sous le contrôle d'un groupe français (Coignet, Givaudan-Lavirotte, Progil, Chimique de Gerland, etc.) ou étrangers (Lumière, Guimet, Novalis, etc.). Sauf Givaudan-Lavirotte passée successivement sous la tutelle de Rhône-Poulenc (1960), Air liquide (1989) et Isaltis (2011) et Gonnet qui a pris le nom de sa marque principale de teintures (Idéal), ces sociétés n'ont pas subsisté comme filiales de groupes qui ont souvent eux-mêmes éclaté ensuite (Kuhlmann, Rhône-Poulenc, etc.). Certaines entreprises spécialisées ont été dépassées par l'évolution technologique. En 1966, le conseil d'administration de Silvestre justifie ainsi la cessation d'exploitation par le « rétrécissement continuel du marché des produits cupriques classiques et la concurrence des produits fongicides de synthèse $»^{24}$. La même année, la fermeture de l'usine Guimet serait rendue nécessaire par son caractère «très onéreux, ne répondant plus aux desideratas d'une production industrielle moderne et efficace $»^{25}$, le groupe anglais repreneur Reckitt se contentant de garder la société française pour la commercialisation de ses produits. Les usines-mères lyonnaises, gagnées par l'urbanisation, ont souvent disparu, à

\footnotetext{
${ }^{24}$ AD Rhône, 3875W841, registre du commerce, TC Lyon, 54B866, rapport du conseil d'administration à l'assemblée générale extraordinaire du 21 juillet 1966.

${ }^{25}$ AD Rhône, 3875W849, registre du commerce, TC Lyon, 55B176, procès-verbal de la séance du conseil d'administration, 22 novembre 1966.
} 
l'exception encore de Givaudan-Lavirotte qui subsiste toujours en pleine ville à proximité du quartier des États-Unis. En périphérie de l'agglomération, les usines de Trux-Mistral à Collonges et de Gignoux à Neuville se sont, en revanche, développées : la première, passée par Le Silicate (1952), Progil (1962), Rhône-Poulenc (1969), serait pour Solvay le leader «mondial des silices précipitées de haute performance, utilisées entre autres dans les pneumatiques automobiles $^{26}$ »; la seconde, «spécialisée dans les synthèses chimiques fines, [...] se prépare également à devenir le $3^{\mathrm{e}}$ pôle européen du groupe [Sanofi] ${ }^{27}$. Du côté des entreprises multiétablissements, si certaines unités de production extérieures subsistent, à l'image pour Progil de Pont-de-Claix, sous la forme d'une plateforme chimique associant plusieurs exploitants, la nouvelle usine implantée par Lumière en 1967 à Saint-Priest pour les papiers photographiques a disparu dès 1996, sous l'enseigne Ilford, avant même l'effondrement complet de la filière argentique.

Des entreprises chimiques moyennes de la région lyonnaise ont donc pu survivre pendant parfois plus d'un siècle de manière indépendante avec des productions de spécialités fines, malgré les moyens limités des familles fondatrices qui les contrôlaient, à côté des grands groupes nationaux. Les années 1960 sont certes marquées par une forte tendance à la concentration et à la restructuration qui semble en sonner le glas. Mais ces grands groupes nationaux ont été, à l'issue notamment de leur privatisation, à leur tour largement démantelés à

\footnotetext{
${ }^{26}$ www.solvay.fr/fr/implantations/collonges-au-mont-dor/index.html (consulté le 26 novembre 2015).

${ }^{27}$ www.sanofi.fr/l/fr/fr/layout.jsp?cnt=78E1307E-31BC-4815-AF98-4778BF224148 (consulté le 26 novembre 2015).
} 
partir des années 1990 ; certaines grandes usines, comme Pont-deClaix ou Roussillon (Isère), sont devenues des plates-formes associant différents opérateurs économiques, qui peuvent être de taille moyenne, même s'ils ne sont plus nécessairement familiaux et indépendants. D'après le syndicat professionnel, la région RhôneAlpes serait, avec près de 500 établissements et 31500 salariés $^{28}$ soit un effectif d'une remarquable stabilité depuis cinquante ans - la première région de production chimique en France, avec une prédominance de PME et d'entreprises de taille intermédiaire (ETI $)^{29}$. Et le passage sous contrôle étranger ne menace pas nécessairement les sites concernés, au contraire.

\footnotetext{
${ }^{28}$ Les dernières statistiques de l'INSEE disponibles donnent même, au 31 décembre 2012, 22124 salariés dans l'industrie chimique et 13245 dans l'industrie pharmaceutique, dont respectivement 9188 et 9576 pour le seul Rhône, non compris la fabrication de produits en caoutchouc en plastique; www.insee.fr (consulté le 26 novembre 2015).

${ }^{29}$ D'après la présentation sur le site de l'Union des industries chimiques RhôneAlpes, $\quad$ www.chimie-rhonealpes.org/Union-des-industries-chimiques-RhoneAlpes/La-Chimie-en-Rhone-Alpes (consulté le 26 novembre 2015). Les chiffres de 1'INSEE sont en fait, au 31 décembre 2012, de 509 établissements industriels pour la chimie et 96 pour la pharmacie, dont respectivement 28 et 20 de plus de deux cents salariés seulement, et 90 et 41 de plus de cinquante.
} 
Tableau 11 : Devenir des entreprises et des usines chimiques étudiées.

\begin{tabular}{|c|c|c|}
\hline Entreprises & Devenir de l'entreprise & Devenir usine lyonnaise \\
\hline Éts Coignet & $\begin{array}{l}\text { 1960, absorption par } \\
\text { Kuhlmann }\end{array}$ & $\begin{array}{l}\text { fermeture années 1960- } \\
1970\end{array}$ \\
\hline Givaudan-Lavirotte & $\begin{array}{l}\text { 1960, filiale Rhône- } \\
\text { Poulenc }\end{array}$ & en activité (groupe Isaltis) \\
\hline Éts Lumière & $\begin{array}{l}\text { 1962, filiale CIBA } \\
\text { (Suisse) }\end{array}$ & fermeture 1976 \\
\hline Chimique de Gerland & $\begin{array}{l}\text { 1989, cession branche } \\
\text { chimique à Elf-Atochem }\end{array}$ & $\begin{array}{l}\text { fermeture années 1970- } \\
1980\end{array}$ \\
\hline Éts Gillet / Progil & $\begin{array}{l}\text { 1969, absorption par } \\
\text { Rhône-Poulenc }\end{array}$ & fermeture $1964 / 1965$ \\
\hline Crème Simon & $\begin{array}{l}\text { 1984, dépôt de bilan, } \\
\text { reprise et transfert à } \\
\text { Figeac (Lot), } 1990 \\
\text { disparition }\end{array}$ & fermeture 1984 \\
\hline Éts Silvestre & 1931, filiale AFC & fermeture 1977 \\
\hline Éts A. Dousselin & 1973, cessation d'activité & fermeture 1966 \\
\hline Trux-Mistral & $\begin{array}{l}\text { 1952, absorption par Le } \\
\text { Silicate, Paris }\end{array}$ & en activité (Solvay) \\
\hline $\begin{array}{l}\text { Lavix Éts Burlaton \& } \\
\text { Richelmy }\end{array}$ & 1961, faillite & fermeture 1961 \\
\hline Laboratoires Novalis & $\begin{array}{l}\text { 1968, prise de contrôle } \\
\text { par American Cyanamid } \\
\text { (USA) }\end{array}$ & fermeture 1995 \\
\hline Gignoux \& $\mathrm{C}^{\mathrm{ie}}$ & $\begin{array}{l}\text { 1953, absorption par } \\
\text { UCLAF }\end{array}$ & en activité (Sanofi) \\
\hline Éts Gonnet & $\begin{array}{l}\text { 2004, filiale groupe AC } \\
\text { Marca (Espagne) }\end{array}$ & $\begin{array}{l}\text { transfert à Vaulx-en-Velin } \\
1982\end{array}$ \\
\hline Éts Guimet & 1965, filiale Reckitt (GB) & fermeture 1967 \\
\hline
\end{tabular}

